\title{
Optical positions of seventeen radio stars from circumzenithal observations by the method of equal altitudes
}

\author{
I. Pešek ${ }^{1}$, J. Hefty ${ }^{2}$ and V. Skoupý ${ }^{3}$ \\ 1 Astronomical Observatory, Czech Technical University in Prague, Thákurova 7, 16629 Praha 6, Czech Republic \\ 2 Astronomical Observatory, Slovak Technical University in Bratislava, Radlinského 11, 81368 Bratislava, Slovak \\ Republic \\ 3 Research Institute of Geodesy, Topography and Cartography, 25066 Zdiby 98, Czech Republic
}

Received November 27; accepted January 2, 1996

\begin{abstract}
Positions and proper motions of seventeen radio stars were improved from observations by the method of equal altitudes as performed by circumzenithals in Prague, Pecný and Bratislava during 1980-1994. Average accuracy of right ascensions, declinations and their proper motions is $0.004,0 . .04,0.001 \mathrm{y}^{-1}$ and $0^{\prime \prime} .01 \mathrm{y}^{-1}$, respectively.
\end{abstract}

Key words: radio continuum: stars astrometry — reference systems

\section{Introduction}

Problem of connecting the optical and radio reference frames, particularly linking the system of the Hipparcos catalog to an inertial frame, represented by the extragalactic radio sources, had caused a necessity of intensive observations of radio stars, the only objects observable by means of the optical and radio astrometry.

In the former Czechoslovakia, three circumzenithals at the Astronomical Observatory of the Czech Technical University in Prague (successively at two observing sites), Geodetic Observatory Pecný in Ondřejov and the Astronomical Observatory of the Slovak Technical University in Bratislava - participated with observations by the method of equal altitudes in the international cooperation to derive the Earth rotation parameters. From 1980, radio stars were gradually included in their observing programs. The FK4 stars were completed by the non-FK4 stars from the Débarbat's (1986) letter to the final number of seventeen. Radio stars have been regularly observed in Prague and Pecný since 1980 and, in Bratislava, were observed in a period of 1987-1993.

The preliminary results from the first ten years of the Prague observations proved the reliability of the method (Pešek 1993). Now we present the improved positions and proper motions as derived in a global adjustment of the data from observations by the three instruments during 1980-1994.

The adjustment can be modified to derive immediately the parameters of mutual orientation of the optical and ra-

Send offprint requests to: I. Pešek dio reference frames provided the coordinates of the radio stars are known in both systems. Due to the lack of positions referred to the extragalactic frame, we are only able to estimate the accuracy, which we can expect for the link based on the discussed data, and cross correlations between the orientation parameters.

\section{Data and the adjustment}

The data to be analysed has been obtained from regular observations of three circumzenithals VÚGTK $100 / 1000 \mathrm{~mm}$ (magnification $100 \times$, zenith distance $30^{\circ}$, typical accuracy of one star transit $\left.0.2^{\prime \prime}-0.3^{\prime \prime}\right)$. The list of the radio stars is shown in Table 1 . Coordinates of the observatories, in the case of Prague before and after 1985, are:

$\begin{array}{lccc} & \text { Code } & \phi & \lambda_{\text {east }} \\ \text { Prague } & \text { PRE } & 50^{\circ} 04^{\prime} 40^{\prime \prime} & 0^{\mathrm{h}} 57^{\mathrm{m}} 40.8^{\mathrm{s}} \\ & \text { PRD } & 50^{\circ} 06^{\prime} 20^{\prime \prime} & 0^{\mathrm{h}} 57^{\mathrm{m}} 33.3^{\mathrm{s}} \\ \text { Pecný } & \text { PYD } & 49^{\circ} 54^{\prime} 56^{\prime \prime} & 0^{\mathrm{h}} 59^{\mathrm{m}} 09.4^{\mathrm{s}} \\ \text { Bratislava } & \text { BRC } & 48^{\circ} 09^{\prime} 18^{\prime \prime} & 1^{\mathrm{h}} 24^{\mathrm{m}} 47.7^{\mathrm{s}}\end{array}$

A typical observing group consists of 28 reference FK4/FK5 stars and one or more radio stars. The absolute terms of the observation equations, $\delta h$ (e.g. Vondrák 1991), were originally referred to the FK4, FK4J2000 and FK5 catalogs in different periods of observations, and they all were recalculated, prior to the present analysis, to be referred to the INCA catalog (Turon et al. 1992). 
Table 1. List of the radio stars with number of observations

\begin{tabular}{|c|c|c|c|c|c|c|c|c|c|}
\hline \multicolumn{5}{|c|}{ Star identification } & \multicolumn{5}{|c|}{ Number of observed west/east transits } \\
\hline INCA & Name & FK & $\mathrm{BD}$ & HD & PRE & PRD & PYD & $\mathrm{BRC}$ & Total \\
\hline 3693 & $\zeta$ And & 27 & +23106 & 4502 & $6 / 20$ & $24 / 29$ & $52 / 50$ & $16 / 16$ & $98 / 115$ \\
\hline 6669 & & 2096 & +22 & 8634 & & $13 / 17$ & $24 / 16$ & $8 / 1$ & $45 / 34$ \\
\hline 14576 & $\beta$ Per & 111 & $+40 \quad 673$ & 19356 & $14 / 15$ & $25 / 37$ & $32 / 37$ & $26 / 15$ & $97 / 104$ \\
\hline 20070 & b Per & & +491150 & 26961 & & $21 / 29$ & $12 / 10$ & $27 / 12$ & $60 / 51$ \\
\hline 27913 & 54 Ori & & +201162 & 39587 & & & & $11 / 11$ & $11 / 11$ \\
\hline 37629 & $\sigma$ Gem & & +291590 & 62044 & & $33 / 5$ & $7 / 17$ & $21 / 26$ & $61 / 48$ \\
\hline 51814 & $37 \mathrm{UMa}$ & 398 & +571277 & 91480 & $23 / 3$ & $31 / 1$ & $0 / 7$ & $19 / 22$ & $73 / 33$ \\
\hline 61281 & $\kappa$ Dra & 472 & $+70 \quad 703$ & 109387 & $3 / 9$ & $33 / 51$ & $70 / 49$ & $20 / 10$ & $126 / 119$ \\
\hline 62512 & & & +611320 & 111456 & & & & $7 / 12$ & $7 / 12$ \\
\hline 66257 & HR 5110 & 502 & +372426 & 118216 & $18 / 22$ & $53 / 43$ & $64 / 56$ & $23 / 23$ & $158 / 144$ \\
\hline 71115 & 26 Boo & 3151 & +222715 & 127739 & & & & $12 / 6$ & $12 / 6$ \\
\hline 79607 & $\sigma \mathrm{CrB} \mathrm{A}$ & & +342750 & 146361 & & $34 / 21$ & $53 / 18$ & $31 / 10$ & $118 / 49$ \\
\hline 92420 & $\beta \mathrm{Lyr}$ & 705 & +333223 & 174638 & $9 / 14$ & $54 / 41$ & $89 / 83$ & $22 / 27$ & $174 / 165$ \\
\hline 94013 & & & +522350 & 179094 & & & & $6 / 13$ & $6 / 13$ \\
\hline 108317 & VV Cep & 3756 & +622007 & 208816 & & $27 / 34$ & $9 / 55$ & $2 / 35$ & $38 / 124$ \\
\hline 113561 & V509 Cas & 3839 & +562923 & 217476 & & $19 / 53$ & $39 / 92$ & $3 / 11$ & $61 / 156$ \\
\hline 116584 & $\lambda$ And & 890 & +454283 & 222107 & $10 / 47$ & $22 / 89$ & $35 / 126$ & $17 / 26$ & $84 / 288$ \\
\hline
\end{tabular}

The method of equal altitudes is based on a principal assumption that the zenith distance is exactly the same for all stars observed in one group. The real observations, however, suffer from systematic errors which appear as deformations of the apparent almucantar of the instrument. These deformations reach to $0.1^{\prime \prime}-0.2^{\prime \prime}$ and change slowly with azimuth $a$ and time $\tau$. They can be approximated by the formula (Pešek 1995)

$$
\begin{array}{r}
D(a, \tau)=\sum_{k=2}^{K}\left\{\left[c_{k}+\sum_{n=1}^{N}\left(c_{\mathrm{s} k n}^{\prime} \sin 2 \pi n \tau+\right.\right.\right. \\
\left.\left.c_{\mathrm{c} k n}^{\prime} \cos 2 \pi n \tau\right)\right] \cos k a+ \\
+\left[s_{k}+\sum_{n=1}^{N}\left(s_{\mathrm{s} k n}^{\prime} \sin 2 \pi n \tau+\right.\right. \\
\left.\left.\left.s_{\mathrm{c} k n}^{\prime} \cos 2 \pi n \tau\right)\right] \sin k a\right\},
\end{array}
$$

where time $\tau=\frac{1}{N}\left(\mathrm{JD}-\mathrm{JD}_{0}\right) / 365.2422$ is expressed in terms of the analysed period of $N$ years from an initial epoch $\mathrm{JD}_{0}$. Parameters $c_{k}, \ldots s_{\mathrm{ckn}}^{\prime}$ are to be estimated from the adjustment. Due to a non uniform distribution and insufficient density of observations, only the main features of the deformations could be modelled in the present analysis. The azimuthal expansions were restricted to the degree of $K=3$ for the Prague and Pecný circumzenithals, and to $K=2$ in the case of Bratislava.

The linearized observation equation is generally a function of four 'group' unknowns $z, \dot{z}$, UT0 - UTC and $\phi$ (zenith distance, its time derivative, clock correction and latitude) of each group, of the deformation parameters $c_{k}$, $\ldots s_{\mathrm{ckn}}^{\prime}$ of all instruments/sites, of the coordinates, $\alpha, \delta$, and proper motions, $\mu_{\alpha}, \mu_{\delta}$, of the stars being improved.
Let us denote the parts corresponding to the group unknowns, deformation parameters and corrections to the star positions as $g, d$ and $s$, respectively, and the residual as $v$. The observation equation for the $j$-th transit will then read

$$
\sum g_{u j}+\sum d_{m j}+\sum s_{w j}=\delta h_{j}+v_{j}
$$

For a particular transit, the only nonzero terms in the above sums are the following: $g_{u}$ - for all transits observed in the $u$-th group, $d_{m}$ - for all transits observed by the $m$-th instrument and, $s_{w}$ - for all transits of the $w$-th radio star.

The part $s$ of the observation equation (Eq. 1) is

$$
s=\left(\mathrm{d} \alpha+\mu_{\alpha} \Delta T\right) \cos \phi \sin a+\left(\mathrm{d} \delta+\mu_{\delta} \Delta T\right) \cos q,
$$

where $q$ is parallactic angle and $\Delta T$ time elapsed from the mean epoch of observation of the respective star. As it can be seen, the coefficients of the unknowns (and also the accuracy of derived star coordinates) depend on azimuth of the transit. As a consequence, we did not estimate declinations of five stars, whose coefficients $|\cos q|$ are less than 0.22 . Different number of east and west transits implies a correlation between $\alpha$ and $\delta$,

$r_{\alpha \delta}=\left(n_{\mathrm{E}}-n_{\mathrm{W}}\right) /\left(n_{\mathrm{E}}+n_{\mathrm{W}}\right)$.

\section{Results and discussions}

The observation equations (Eq. 1) yield an extensive system of normal equations. The group unknowns can however be easily separated, so that a much smaller system for the star coordinates, proper motions and almucantar deformations is to be solved in the adjustment. 
Table 2. Radio star optical positions and centennial proper motions as derived from circumzenithal observations (mean epoch of observations, equator and equinox J2000.0, reference catalog INCA)

\begin{tabular}{|c|c|c|c|c|c|c|c|c|c|}
\hline \multicolumn{6}{|c|}{ Positions and proper motions } & \multicolumn{4}{|c|}{ Mean errors } \\
\hline INCA & $\alpha$ & $\mu_{\alpha}$ & $\delta$ & $\mu_{\delta}$ & Epoch & $\sigma(\alpha)$ & $\sigma\left(\mu_{\alpha}\right)$ & $\sigma(\delta)$ & $\sigma\left(\mu_{\delta}\right)$ \\
\hline 3693 & $0^{\mathrm{h}} 47^{\mathrm{m}} 20^{\mathrm{s}} .429$ & -0.672 & $24^{\circ} 16^{\prime} 02^{\prime \prime} .80$ & $-9^{\prime \prime} 56$ & 1987.15 & 0.003 & 0.086 & 0.02 & 0.57 \\
\hline 6669 & 12535.652 & -0.073 & 233041.75 & -1.07 & 1989.30 & 0.006 & 0.181 & 0.04 & 1.07 \\
\hline 14576 & 30810.128 & 0.103 & 405720.34 & -0.68 & 1987.84 & 0.002 & 0.054 & 0.04 & 1.00 \\
\hline 20070 & 41814.564 & 0.481 & 501744.56 & - & 1990.03 & 0.003 & 0.161 & 0.09 & - \\
\hline 27913 & 55423.096 & - & 201635.16 & - & 1990.81 & 0.014 & - & 0.07 & - \\
\hline 37629 & 74318.681 & 0.462 & 285302.63 & -23.89 & 1990.51 & 0.003 & 0.167 & 0.03 & 1.73 \\
\hline 51814 & 103509.596 & 0.779 & - & - & 1987.80 & 0.003 & 0.088 & - & - \\
\hline 61281 & 123329.072 & -1.305 & $6947 \quad 17.58$ & 5.23 & 1988.08 & 0.004 & 0.095 & 0.07 & 1.67 \\
\hline 62512 & 124839.323 & - & - & - & 1990.97 & 0.009 & - & - & - \\
\hline 66257 & 133447.720 & 0.735 & 371056.86 & 0.92 & 1987.35 & 0.002 & 0.043 & 0.03 & 0.68 \\
\hline 71115 & 143232.597 & - & 221535.55 & - & 1991.43 & 0.013 & - & 0.04 & - \\
\hline 79607 & 161441.052 & -2.237 & 335131.93 & -7.71 & 1990.63 & 0.003 & 0.111 & 0.04 & 1.49 \\
\hline 92420 & 185004.795 & -0.012 & 332145.69 & -0.23 & 1987.67 & 0.002 & 0.043 & 0.02 & 0.57 \\
\hline 94013 & 190825.881 & - & - & - & 1991.09 & 0.008 & - & - & - \\
\hline 108317 & 215639.152 & 0.005 & - & - & 1990.27 & 0.003 & 0.144 & - & - \\
\hline 113561 & $2300 \quad 05.103$ & 0.191 & - & - & 1989.01 & 0.002 & 0.069 & - & - \\
\hline 116584 & 233733.657 & 1.553 & 462734.41 & -41.04 & 1987.72 & 0.002 & 0.050 & 0.04 & 1.19 \\
\hline
\end{tabular}

Contribution of individual instruments to the final solution depends on number of observations and their internal accuracy. Dispersions of the results, derived for each instrument separately, from the results of the common solution, $\sigma^{2}=\sum\left[(\Delta \alpha \cos \delta)^{2}+\Delta \delta^{2}\right] / n$ ( $n$ is the number of radio stars observed by the respective instrument), yield the weights $0.77,1.00$ and 0.59 for Prague, Pecný and Bratislava, respectively.

Adjusted coordinates and centennial proper motions of all seventeen radio stars and corresponding mean errors are listed in Table 2. The positions are referred to the mean epochs of observations, equator and equinox J2000, and to the INCA catalog.

Table 3. Comparison with Bordeaux automatic meridian circle (in the sense 'circumzenithals minus meridian circle'). INCA values were used for proper motions not listed in Table 2. [ $\Delta \alpha$ in $0.001, \Delta \delta$ in $\left.0^{\prime \prime} .01\right]$

\begin{tabular}{rrrrrr}
\hline INCA & $\Delta \alpha$ & $\Delta \delta$ & INCA & $\Delta \alpha$ & $\Delta \delta$ \\
\hline 3693 & +4 & -16 & 66257 & +3 & -4 \\
6669 & +2 & -1 & 71115 & -23 & -32 \\
14576 & +3 & +2 & 79607 & +3 & +5 \\
20070 & +5 & +20 & 92420 & +1 & +1 \\
27913 & +10 & +17 & 94013 & -8 & - \\
37629 & +2 & -13 & 108317 & 0 & - \\
51814 & +5 & - & 113561 & -4 & - \\
61281 & +1 & +9 & 116584 & -2 & 0 \\
62512 & -4 & - & & & \\
\hline
\end{tabular}

Mean errors of four stars, namely $27913,51814,71115$ and 94013 , exceed substantially the average error level of 0 s.004 and 0.'04 in right ascension and declination, obviously due to a small number of observations during a short period of only six years. We cannot explain the insufficient accuracy of proper motion of 20070 which was very often observed during a whole period of observations. Consequently, we have omitted proper motions of those stars in the Table.

To check the external accuracy, we compared our results with positions obtained from observations on the Bordeaux automatic meridian circle (Réquième \& Mazurier 1991). The differences listed in Table 3 show a very good agreement of both results; only the differences in declination of 3693 and 71115 exceed slightly the correponding mean errors of $0{ }^{\prime \prime} 14$ and 0.22 , respectively.

The $79607(\sigma \mathrm{CrB} \mathrm{A})$ is the brighter component of a close binary with the components separated by only $5^{\prime \prime}$. Thus we could expect a greater uncertainty in its position, since observers tend to coincide images of the photocenter rather than those of the primary. This suspicion was, however, disproved not only by small internal errors but also by a good agreement with the Bordeaux position.

\section{Expected accuracy of link to the extragalactic frame}

The procedure described above gives positions of individual stars. Provided the radio star coordinates are known in both optical and extragalactic system, the adjustment can be easily modified to derive immediately six parameters of mutual orientation of the two reference frames. 
According to Lindegren \& Kovalevsky (1995) notation, the parameters are three rotations $\varepsilon_{i}$ around axes of optical rectangular equatorial system and their time derivatives $\omega_{i}=\dot{\varepsilon}_{i}$. When

$$
\begin{aligned}
\mathrm{d} \alpha+\mu_{\alpha} \Delta T & =\left(\varepsilon_{x}+\omega_{x} \Delta T\right) \cos \alpha \tan \delta+ \\
& +\left(\varepsilon_{y}+\omega_{y} \Delta T\right) \sin \alpha \tan \delta-\left(\varepsilon_{z}+\omega_{z} \Delta T\right), \\
\mathrm{d} \delta+\mu_{\delta} \Delta T & =-\left(\varepsilon_{x}+\omega_{x} \Delta T\right) \sin \alpha+\left(\varepsilon_{y}+\omega_{y} \Delta T\right) \cos \alpha
\end{aligned}
$$

are assigned to Eq. (2), observation equations can be solved for the orientation parameters.

For the moment we do not have reliable star coordinates and proper motions referred to the extragalactic frame. Thus we could only estimate the expected internal accuracy of $\varepsilon_{i}, \omega_{i}$ and cross correlations between the unknowns as shown in Table 4.

Table 4. Expected accuracy of the orientation parameters from circumzenithal observations

\begin{tabular}{llrrrrrr}
\hline & mean & \multicolumn{7}{c}{ correlation } \\
& error & $\varepsilon_{x}$ & $\varepsilon_{y}$ & $\varepsilon_{y}$ & $\omega_{x}$ & $\omega_{y}$ & $\omega_{z}$ \\
\hline$\varepsilon_{x}$ & $0^{\prime \prime} 010$ & 1.0 & & & & & \\
$\varepsilon_{y}$ & 0.012 & 0.2 & 1.0 & & & & \\
$\varepsilon_{z}$ & 0.012 & 0.2 & -0.1 & 1.0 & & & \\
$\omega_{x}$ & $0^{\prime \prime} 002 \mathrm{y}^{-1}$ & 0.4 & 0.1 & 0.1 & 1.0 & & \\
$\omega_{y}$ & 0.003 & 0.1 & 0.4 & 0.0 & 0.2 & 1.0 & \\
$\omega_{z}$ & 0.003 & 0.1 & 0.0 & 0.4 & 0.1 & -0.2 & 1.0 \\
\hline
\end{tabular}

\section{Conclusions}

Up to seventeen radio stars were regularly observed by the method of equal altitudes by circumzenithals VÚGTK
100/1000 mm at observatories in Prague, Pecný and Bratislava during a period 1980-1994. The data was homogenized by transforming to the INCA catalog. Systematic errors of various origin appear as deformations of the apparent almucantar of the instrument. Parameters of the deformation models were derived along with coordinates and proper motions of the radio stars in a global adjustment. The star positions are referred to the mean epochs of observations and to the equator and equinox J2000. Average mean errors in right ascensions, declinations and their proper motions are 0.004, 0.'04, 0.001 $\mathrm{y}^{-1}$ and $00^{\prime \prime} 01 \mathrm{y}^{-1}$, respectively.

The data can also be used to derive directly the mutual orientation of two reference frames, e.g. optical and extragalactic, provided the radio star positions are known in both systems. For the moment we could only estimate the expected accuracy of orientation angles $\varepsilon_{i}$ and their rates $\omega_{i}$ to be 0. ! 01 and $0 . \prime 003 \mathrm{y}^{-1}$.

Acknowledgements. The authors wish to express appreciation to all colleagues, who participated as the observers in collecting the data, for their extended effort during a whole period of observations. The support of grant of the Czech Technical University No. 10018244 is also very appreciated.

\section{References}

Débarbat S., 1986, IAU WG Comm. Lett. (unpubl.)

Lindegren L., Kovalevsky J., 1995, A\&A 304, 189

Pešek I., 1993, A\&AS 97, 777

Pešek I., 1995, Acta Polytech. 35/2, 5

Réquième Y., Mazurier J.M., 1991, A\&AS 89, 311

Turon C., et al., 1992, The Hipparcos Input Catalogue, ESA

Vondrák J., 1991, Bull. Astron. Inst. Czechosl. 42, 283 